Original Research Article

\title{
Self-medication practices among medical and nursing students in Tripura, India: a mixed-method study
}

\author{
Nabarun Karmakar, Tamal Chakraborty*, Anjan Datta, Kaushik Nag, Shib Sekhar Datta
}

Department of Community Medicine, Tripura Medical College and Dr. BRAM Teaching Hospital, Hapania, P.O. - ONGC,

Agartala, Tripura, India

Received: 04 December 2018 Accepted: 29 December 2018

\section{*Correspondence to: \\ Dr. Tamal Chakraborty, Email: drtamalchakraborty@ gmail.com}

Copyright: (C) the author(s), publisher and licensee Medip Academy. This is an openaccess article distributed under the terms of the Creative Commons Attribution NonCommercial License, which permits unrestricted noncommercial use, distribution, and reproduction in any medium, provided the original work is properly cited.

\begin{abstract}
Background: In developing countries like India, self-medication is a common practice because of costly clinical services and easy availability of medicine from pharmacies. There is an increase trend of self-medication among different health professional students apart from general people. This study aims to assess selfmedication practice among medical and nursing students in Tripura, India.

Methods: This cross-sectional study was conducted among undergraduate medical and nursing students of Tripura Medical College and Tripura College of Nursing, of Tripura for two months (January- February 2018). A pre-tested, semistructured schedule was used to collect the required information. The collected data was entered in SPSS version 16.0, represented in proportions and $\mathrm{p}<0.05$ was considered as statistically significant.

Results: Overall 353 (88.3\%) students of both courses practiced self-medication; prevalence was more among medical $(91.5 \%)$ than nursing students $(85 \%)$. Main source of information among medical students were books $(90.2 \%)$ while $56.5 \%$ nursing students replied friends and/or seniors as primary source. Majority (53\%) respondents took self-medication for headache $(79.2 \%$ medical vs $72.4 \%$ nursing students) followed by fever (77.6\% medical vs $52.4 \%$ nursing). Less than half of the respondents ( $48.5 \%$ medical vs $46.5 \%$ nursing students) believed that selfmedication was harmful; maximum nursing students $(71.5 \%)$ believed that selfmedication is self-care as compare to medical students $(56.5 \%)$.

Conclusions: The study found wide practice of self-medication among students, inappropriate use of which may cause serious health hazards. Promoting education regarding self-medication and making healthcare system available can reduce self-medication practices substantially.
\end{abstract}

Keywords: Books, Friends, Headache, India, Self-medication

\section{INTRODUCTION}

Medication plays a vital role in health care delivery, being an important therapeutic tool in the hands of health professionals. ${ }^{1}$ Even general people throughout world, act on their own for their health by practicing selfmedication. $^{2}$ WHO defines Self- medication as selection and use of medicines by individuals to treat recognized illness or symptoms. ${ }^{3}$ This includes acquiring medicines without prescription, resubmitting old prescriptions, sharing medicines with relatives/friends or using leftover medicines stored at home..$^{4-7}$ It also includes medication for family members, especially for children or elderly. ${ }^{8}$
Self-medication with OTC medicines is known as responsible self-medication to distinguish from practice of purchasing and using a prescription medicine without a doctor's prescription. ${ }^{9}$ Practice of responsible selfmedication is generally considered as safe and effective worldwide; but prescribed drugs dispensed without valid prescriptions in India is a pitfall in this regard may poses many medical and social challenges. ${ }^{10,11}$ WHO is promoting practice of self-medication for effective and quick relief of symptoms to reduce burden on health services. ${ }^{12}$ But irrational drug use can cause wastage of resources, increased resistance of pathogens, even serious 
health hazards. ${ }^{13}$ Self-medication defers from self-care in that it involves drugs that may do good or cause harm. ${ }^{14}$

In developing countries like India, self-medication is common because of costly clinical services and easy alternatives like pharmacies being first point of contact for patient. ${ }^{15}$ Pharmacists and pharmacy attendants also play role in fostering self-medication among the public. ${ }^{12}$ Even, medical and paramedical students are commonly involved, without complete knowledge about therapy. ${ }^{16}$ Literature reported increase trends of self-medication among different health professional students (prevalence ranging from $57.7 \%$ to $76 \%$ ) who gain knowledge of drugs during their course as compared to others. ${ }^{8,17,18}$

Though studies were conducted both at community as well as institutional level but there is paucity of literature in this part of the country. With this background this study was conducted among undergraduate medical and nursing students of West Tripura district, Tripura to assess their self-medication practices, compare and also to focus on the awareness and attitude of students who practice selfmedication.

\section{METHODS}

This institutional based cross-sectional study was carried out among medical and nursing students of one medical (Tripura Medical College and Dr. BRAM Teaching Hospital, Hapania, Agartala) and one nursing (Tripura College of Nursing, Hapania, Agartala) institution of West Tripura District, Tripura for a period of two month, January- February 2018. Sample size was calculated using formula $=Z \alpha^{2} p q$ / L2 where, $p=$ prevalence; $q=100-p$; $\mathrm{L}=$ allowable error (At $95 \% \mathrm{CI}, \mathrm{Z}_{\alpha}=1.96$ ). Taking $67 \%$ as prevalence of self-medication, with $10 \%$ relative allowable error and an additional $10 \%$ increase to compensate for any non-response sample size calculated was $200 .{ }^{19}$ Finally, 200 medical and 200 nursing students were interviewed randomly from each institution using the list of all students from four professional years as sampling frame. Students available on the day of data collection and willing to participate were taken in this study.

A pre-designed, pre-tested, semi-structured questionnaire having two parts:

- Part I: socio-demographic characteristics and

- Part II: questions related to self-medication were used to collect information.

The questionnaire was prepared considering previous literature, applied to 40 students different from study participants, and all ambivalent and unclear questions were rephrased or removed ${ }^{4,6,7,19}$ Students were selected randomly after entering and interviewed after obtaining informed consent. Collected data was entered into SPSS version 16.0 and represented in frequency, percentages in tables and diagram. Significance were assessed using
Pearson's Chi-square test ( $\mathrm{p}<0.05$ was considered as significant).

\section{Qualitative method (focus group discussion)}

Two semi-structured focus group discussions (FGDs) as per PRIA (Participatory Research in Asia) guidelines were facilitated by faculties to explore issues pertinent to selfmedication among medical and nursing students. Each FGD was conducted for about 45 minutes till point of exhaustion. Those students who talked freely and communicated effectively during quantitative data collection were chosen for FGDs.

The FGDs started with briefing them about the purpose of interview, discussions were audio recorded and transcripts were prepared as verbatim on the same day. Descriptive coding was done in transcripts thorough manual content analysis. Later, similar codes were merged together to form the categories or themes which were reviewed by another researcher who was not involved in earlier stage to avoid subjective bias and strengthen interpretive credibility. A statement in 'Italics and inverted comma' depicts direct quotes/words/statements from participants.

\section{Ethical consideration}

This research study was approved by institutional ethics committee (Tripura Medical College and Dr. BRAM Teaching Hospital, Agartala).

\section{RESULTS}

This institution based cross-sectional study was conducted among 400 students (200 medical and 200 nursing), where majority $(64.5 \%)$ undergraduate medical students were $<22$ years old with a mean age of $20.9 \pm 1$.3years (1824years) whereas $61.5 \%$ undergraduate B.Sc nursing students were $\geq 22$ years with a mean age of $22.0 \pm 2$.0years (18-26 years). Male students were more in number $(59 \%)$ among medical students; female predominance $(79 \%)$ was among nursing students. Most of them were Hindu (95\% medical vs $92 \%$ nursing students); $58 \%$ medical vs $44 \%$ nursing students belonged to general caste, $10 \%$ ST from both streams. Maximum were from nuclear families ( $87.5 \%$ medical vs $76.5 \%$ nursing students); $84 \%$ medical as compared to $48 \%$ nursing students belonged to upper class as per B.G. Prasad scale updated for $2017 .{ }^{20}$

Figure 1 showed that prevalence of self-medication was more among medical students than nursing students; 183 $(91.5 \%)$ and $170(85 \%)$ respectively practice selfmedication. Overall among 400 students, $353(88.3 \%)$ students of both courses practiced self-medication.

Table 1 showed that, main sources of information for selfmedication among medical students were books (90.2\%), followed by internet $(47.5 \%)$, advertisements in $\mathrm{TV}$, magazines $(44.8 \%)$, guardian $(25.1 \%)$, friends and/or seniors $(19.1 \%)$, previous prescriptions $(9.8 \%)$. Out of 170 
nursing students practising self-medication, 96 (56.5\%) replied friends and/or seniors as primary sources followed by advertisements in TV, magazines (48.2\%), textbooks $(47.1 \%)$, internet $(43.5 \%)$, guardians $(9.4 \%)$, previous prescription $(4.1 \%)$. More nursing students (26.5\%) practiced self-medication with advice from pharmacist and/ or chemists as compared to medical students $(5.5 \%)$.
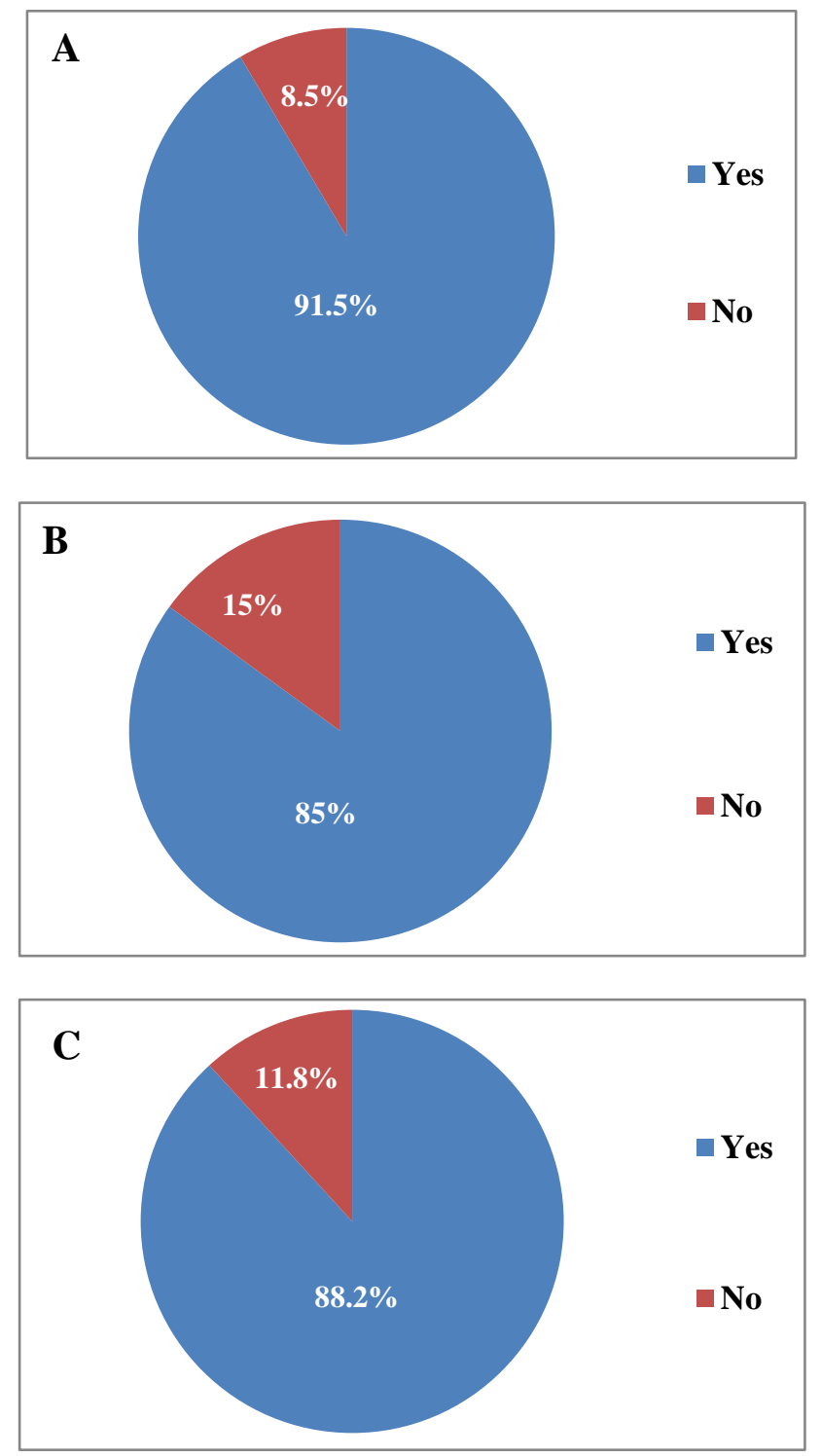

\section{Figure 1: Distribution of participants according to prevalence of self-medication. (A): self-medication among medical students, (B): self-medication among nursing students, $(\mathrm{C})$ : self-medication among all students.}

The reasons for taking self-medication were selfconfidence and awareness about medicine $(62.3 \%$ medical vs $15.3 \%$ nursing students), lack of time to visit a doctor ( $7.6 \%$ medical vs $41.2 \%$ nursing), reluctance to spend money on doctors' fee and lab investigations $(5.5 \%$ medical vs $12.9 \%$ nursing), quick relief ( $22.4 \%$ medical vs $10.6 \%$ nursing). However, students $(49.7 \%$ medical vs $30 \%$ nursing) having previous experience with a disease (themselves or family/friends) took self-medication which got cured previously with same medication.

More than half $(53 \%)$ of the respondents took selfmedication for headache $(79.2 \%$ medical vs $72.4 \%$ nursing students) followed by fever ( $77.6 \%$ medical vs $52.4 \%$ nursing), common cold ( $72.7 \%$ medical vs $49.4 \%$ nursing). Few female students also practice self-medication for their menstrual problems (4.4\% medical vs $2.4 \%$ nursing).

Most common drugs used for self-medication were vitamins and antioxidants $(67.8 \%)$ followed by antibiotics $(61.2 \%)$ and cough syrup (55.9\%) followed by vitamins and antioxidants $(51.8 \%)$ for medical and nursing students respectively. Others were analgesics $(23.5 \%$ medical vs $20.6 \%$ nursing students), anta-acids (37.2\% medical vs $22.6 \%$ nursing students), antipyretics (42.6\% medical vs $34.7 \%$ nursing students), and antispasmodic (6\% medical vs $2.4 \%$ nursing students), anti-allergies (7.6\% medical vs $5.9 \%$ nursing students) and topical preparations (7.6\% medical vs $4.7 \%$ nursing students). Most of the medical students $(87.4 \%)$ knew drug dosage, whereas only $38.2 \%$ nursing students knew about this. Majority medical students $\{122(66.7 \%)\}$ completed course of drug as compared to nursing students $\{75(44.1 \%)\}$. Maximum medical students $(70.5 \%)$ had knowledge about side effects, whereas half $(50.6 \%)$ of the nursing students knew about side effects of drugs; only $\{25(13.5 \%)\}$ medical students experienced drug related adverse effects while 90 $(52.9 \%)$ nursing students experienced side effects. Majority (58.5\% medical vs $45.3 \%$ nursing students) had withdrawn medication after cessation symptoms. Most of the respondents from both streams preferred allopathic medicine (87.4\% medical vs $85.9 \%$ nursing students). More nursing students $\{46(27.1 \%)$ and 28 (16.5\%) respectively preferred Homoeopathy and Ayurveda as compared to medical students $\{35(19.1 \%)$ and $10(5.5 \%)$ respectively\}.

Table 2 showed attitude of participants towards selfmedication. Less than half $(48.5 \%$ medical vs $46.5 \%$ nursing students) believed that self-medication was harmful; maximum nursing students $(71.5 \%)$ believed this as self-care than medical students $(56.5 \%)$. Surprisingly when asked about continuation, only $21 \%$ nursing and $32.5 \%$ medical students reported to continue with selfmedication in the future also. A vast majority of the nursing students $(55.5 \%)$ had a positive attitude toward giving/taking self-medication advice to/from others than medical students $(24.5 \%)$. The opinion of students to curb growing trend of self-medication showed that majority (69.5\% medical vs $62 \%$ nursing students) vouched for preventing the sale/supply of medicines without valid prescription; some others also suggested enforcement of stringent regulations regarding misleading pharmaceutical advertising ( $81.52 \%$ medical vs $72 \%$ nursing students), making health facilities affordable and easily available ( $86.5 \%$ medical vs $89.5 \%$ nursing students) and spreading awareness about adverse effects (86\% medical vs $85 \%$ nursing students). 
Table 1: Distribution of participants according to the characteristics of practice of self-medication.

\begin{tabular}{|c|c|c|c|c|}
\hline \multicolumn{2}{|l|}{ Characteristics } & \multirow{2}{*}{$\begin{array}{l}\text { Frequency }(\%) \text { n } \\
(\%) \\
\text { Medical* }\end{array}$} & \multirow{2}{*}{$\begin{array}{l}\text { Frequency }(\%) \mathbf{n} \\
(\%) \\
\text { Nursing** }\end{array}$} & \multirow{2}{*}{$\begin{array}{l}\text { Frequency }(\%) \text { n } \\
(\%) \\
\text { All Students }^{\wedge *}\end{array}$} \\
\hline & & & & \\
\hline \multirow{7}{*}{$\begin{array}{l}\text { Source of } \\
\text { information } * * *\end{array}$} & Friends and/ or, Seniors & $35(19.1)$ & $96(56.5)$ & $131(37.1)$ \\
\hline & Pharmacist and / or Chemists & $10(5.5)$ & $45(26.5)$ & $55(15.6)$ \\
\hline & Internet & $87(47.5)$ & $74(43.5)$ & $161(45.6)$ \\
\hline & Advertisement in TV, Magazine & $82(44.8)$ & $82(48.2)$ & $164(46.5)$ \\
\hline & Text-Books & $165(90.2)$ & $80(47.1)$ & $245(69.4)$ \\
\hline & Previous prescriptions & $18(9.8)$ & $7(4.1)$ & $25(7.1)$ \\
\hline & Guardian & $46(25.1)$ & $16(9.4)$ & $62(17.6)$ \\
\hline \multicolumn{5}{|c|}{ Reasons for not consulting a doctor ${ }^{* * *}$} \\
\hline \multicolumn{2}{|c|}{ Lack of time to visit a doctor } & $14(7.6)$ & $70(41.2)$ & $84(23.8)$ \\
\hline \multicolumn{2}{|c|}{$\begin{array}{l}\text { Reluctance to spend money on doctors' fee and lab } \\
\text { investigations }\end{array}$} & $10(5.5)$ & $22(12.9)$ & $32(9.1)$ \\
\hline \multicolumn{2}{|c|}{ Self-confidence regarding awareness about medicine } & $114(62.3)$ & $26(15.3)$ & $140(39.7)$ \\
\hline \multicolumn{2}{|c|}{ Previous experience with disease } & $91(49.7)$ & $51(30.0)$ & $142(40.2)$ \\
\hline \multicolumn{2}{|c|}{ Quick relief } & $41(22.4)$ & $18(10.6)$ & $59(16.7)$ \\
\hline \multirow{6}{*}{$\begin{array}{l}\text { Symptoms for } \\
\text { which drugs } \\
\text { were taken*** }\end{array}$} & Fever & $142(77.6)$ & $89(52.4)$ & $231(65.4)$ \\
\hline & Headache & $145(79.2)$ & $123(72.4)$ & $268(75.9)$ \\
\hline & Common Cold and Cough & $133(72.7)$ & $84(49.4)$ & $217(61.5)$ \\
\hline & Gastrointestinal problems & $25(13.7)$ & $53(31.2)$ & $78(22.1)$ \\
\hline & Skin problem and allergies & $11(6.0)$ & $16(9.4)$ & $27(7.6)$ \\
\hline & Menstrual Problems & $8(4.4)$ & $4(2.4)$ & $12(3.4)$ \\
\hline \multirow{9}{*}{$\begin{array}{l}\text { Drug taken for } \\
\text { self- } \\
\text { medication*** }\end{array}$} & Analgesic & $43(23.5)$ & $35(20.6)$ & $78(22.1)$ \\
\hline & Antipyretics & $78(42.6)$ & $59(34.7)$ & $137(38.8)$ \\
\hline & Antibiotics & $112(61.2)$ & $68(40.0)$ & $180(50.9)$ \\
\hline & Cough syrups & $83(45.4)$ & $95(55.9)$ & $178(50.4)$ \\
\hline & Antacids & $68(37.2)$ & $38(22.6)$ & $106(30.02)$ \\
\hline & Antiallergics & $14(7.6)$ & $10(5.9)$ & $24(6.8)$ \\
\hline & Vitamins and antioxidants & $124(67.8)$ & $88(51.8)$ & $212(60.1)$ \\
\hline & Antispasmodics & $11(6.0)$ & $4(2.4)$ & $15(4.2)$ \\
\hline & Topical preparations & $14(7.6)$ & $8(4.7)$ & $22(6.2)$ \\
\hline \multicolumn{5}{|c|}{ Checking of instructions on the medicine } \\
\hline \multicolumn{2}{|l|}{ Yes } & $150(82.0)$ & $88(51.8)$ & $238(67.4)$ \\
\hline \multicolumn{2}{|l|}{ No } & $33(18.0)$ & $82(48.2)$ & $115(32.6)$ \\
\hline \multicolumn{5}{|c|}{ Knowledge about dosage of drug } \\
\hline \multicolumn{2}{|c|}{ Yes } & $160(87.4)$ & $65(38.2)$ & $225(63.7)$ \\
\hline \multicolumn{2}{|l|}{ No } & $23(12.6)$ & $105(61.8)$ & $128(36.3)$ \\
\hline \multicolumn{5}{|c|}{ Completion of course of the drug } \\
\hline \multicolumn{2}{|l|}{ Yes } & $122(66.7)$ & $75(44.1)$ & $197(55.8)$ \\
\hline No & & $61(33.3)$ & $95(55.9)$ & $156(44.2)$ \\
\hline Knowledge abo & it the side effects of drug & & & \\
\hline Yes & & $129(70.5)$ & $86(50.6)$ & $215(60.1)$ \\
\hline No & & $54(29.5)$ & $84(49.4)$ & $138(39.1)$ \\
\hline Experience of a & Iverse effects & & & \\
\hline Yes & & $25(13.5)$ & $90(52.9)$ & $115(32.6)$ \\
\hline No & & $158(86.5)$ & $80(47.1)$ & $238(67.4)$ \\
\hline Withdrawal of & nedication after cessation of sympt & & & \\
\hline Yes & & $107(58.5)$ & $77(45.3)$ & $184(52.1)$ \\
\hline No & & $76(41.5)$ & $93(54.7)$ & $169(47.9)$ \\
\hline Type of & Allopathy & $160(87.4)$ & $146(85.9)$ & $306(86.7)$ \\
\hline treatment & Ayurvedic & $10(5.5)$ & $28(16.5)$ & $38(10.8)$ \\
\hline $\begin{array}{l}\text { modality } \\
\text { taken*** }\end{array}$ & Homeopathy & $35(19.1)$ & $46(27.1)$ & $81(22.9)$ \\
\hline
\end{tabular}


Table 2: Distribution of participants according to their perception and attitude towards self-medication.

\begin{tabular}{|c|c|c|c|c|c|c|c|c|c|}
\hline \multirow[t]{2}{*}{ Item } & \multicolumn{3}{|c|}{ Medical (n=200) } & \multicolumn{3}{|c|}{ Nursing $(n=200)$} & \multicolumn{3}{|c|}{ All Students $(n=400)$} \\
\hline & $\begin{array}{l}\text { Agree n } \\
(\%)\end{array}$ & $\begin{array}{l}\text { Disagree } \\
\text { n }(\%)\end{array}$ & $\begin{array}{l}\text { No } \\
\text { response } \\
\text { n }(\%)\end{array}$ & $\begin{array}{l}\text { Agree } \\
\text { n (\%) }\end{array}$ & $\begin{array}{l}\text { Disagree } \\
\text { n }(\%)\end{array}$ & $\begin{array}{l}\text { No } \\
\text { response } \\
\text { n }(\%)\end{array}$ & $\begin{array}{l}\text { Agree } \\
\text { n (\%) }\end{array}$ & $\begin{array}{l}\text { Disagree } \\
\text { n }(\%)\end{array}$ & $\begin{array}{l}\text { No } \\
\text { response } \\
\text { n }(\%)\end{array}$ \\
\hline $\begin{array}{l}\text { Self-medication is } \\
\text { harmful }\end{array}$ & $\begin{array}{l}97 \\
(48.5) \\
\end{array}$ & $\begin{array}{l}58 \\
(29.0) \\
\end{array}$ & $\begin{array}{l}45 \\
(22.5)\end{array}$ & $\begin{array}{l}93 \\
(46.5)\end{array}$ & $\begin{array}{l}32 \\
(16.0)\end{array}$ & $\begin{array}{l}75 \\
(37.5) \\
\end{array}$ & $\begin{array}{l}190 \\
(47.5)\end{array}$ & $\begin{array}{l}90 \\
(22.5) \\
\end{array}$ & $\begin{array}{l}120 \\
(30.0) \\
\end{array}$ \\
\hline $\begin{array}{l}\text { Self-medication is } \\
\text { a part of self-care }\end{array}$ & $\begin{array}{l}113 \\
(56.5)\end{array}$ & $\begin{array}{l}48 \\
(24.0)\end{array}$ & $\begin{array}{l}39 \\
(19.5) \\
\end{array}$ & $\begin{array}{l}143 \\
(71.5)\end{array}$ & $\begin{array}{l}25 \\
(12.5)\end{array}$ & $\begin{array}{l}32 \\
(16.0)\end{array}$ & $\begin{array}{l}256 \\
(64.0) \\
\end{array}$ & $\begin{array}{l}73 \\
(18.3) \\
\end{array}$ & $\begin{array}{l}71 \\
(17.7)\end{array}$ \\
\hline $\begin{array}{l}\text { Continuation of } \\
\text { self medication }\end{array}$ & $\begin{array}{l}65 \\
(32.5)\end{array}$ & $\begin{array}{l}62 \\
(31.0)\end{array}$ & $\begin{array}{l}73 \\
(36.5)\end{array}$ & $\begin{array}{l}42 \\
(21.0)\end{array}$ & $\begin{array}{l}50 \\
(25.0)\end{array}$ & $\begin{array}{l}108 \\
(54.0)\end{array}$ & $\begin{array}{l}107 \\
(26.7)\end{array}$ & $\begin{array}{l}112 \\
(28.0)\end{array}$ & $\begin{array}{l}181 \\
(45.3)\end{array}$ \\
\hline $\begin{array}{l}\text { Give and take } \\
\text { self-medication } \\
\text { advice from } \\
\text { friends and family } \\
\text { members }\end{array}$ & $\begin{array}{l}49 \\
(24.5)\end{array}$ & $\begin{array}{l}80 \\
(40.0)\end{array}$ & $\begin{array}{l}71 \\
(35.5)\end{array}$ & $\begin{array}{l}111 \\
(55.5)\end{array}$ & $\begin{array}{l}68 \\
(34.0)\end{array}$ & $\begin{array}{l}21 \\
(10.5)\end{array}$ & $\begin{array}{l}162 \\
(40.5)\end{array}$ & $\begin{array}{l}148 \\
(37.0)\end{array}$ & $\begin{array}{l}92 \\
(23.0)\end{array}$ \\
\hline $\begin{array}{l}\text { Prevent medicine } \\
\text { supply without } \\
\text { valid prescription }\end{array}$ & $\begin{array}{l}139 \\
(69.5)\end{array}$ & $\begin{array}{l}18 \\
(9.0)\end{array}$ & $\begin{array}{l}43 \\
(21.5)\end{array}$ & $\begin{array}{l}124 \\
(62.0)\end{array}$ & $\begin{array}{l}61 \\
(30.5)\end{array}$ & $\begin{array}{l}15 \\
(7.5)\end{array}$ & $\begin{array}{l}263 \\
(65.7)\end{array}$ & $\begin{array}{l}79 \\
(19.8)\end{array}$ & $\begin{array}{l}58 \\
(14.5)\end{array}$ \\
\hline $\begin{array}{l}\text { Enforcement of } \\
\text { strict rules }\end{array}$ & $\begin{array}{l}163 \\
(81.5)\end{array}$ & $\begin{array}{l}5 \\
(2.5)\end{array}$ & $\begin{array}{l}32 \\
(16.0)\end{array}$ & $\begin{array}{l}144 \\
(72.0)\end{array}$ & $\begin{array}{l}36 \\
(18.0)\end{array}$ & $\begin{array}{l}20 \\
(10.0)\end{array}$ & $\begin{array}{l}307 \\
(76.7)\end{array}$ & $\begin{array}{l}41 \\
(10.3)\end{array}$ & $\begin{array}{l}52 \\
(13.0) \\
\end{array}$ \\
\hline $\begin{array}{l}\text { Making health } \\
\text { services affordable }\end{array}$ & $\begin{array}{l}173 \\
(86.5) \\
\end{array}$ & $\begin{array}{l}3 \\
(1.5)\end{array}$ & $\begin{array}{l}24 \\
(12.0) \\
\end{array}$ & $\begin{array}{l}179 \\
(89.5)\end{array}$ & $\begin{array}{l}14 \\
(7.0)\end{array}$ & $\begin{array}{l}7 \\
(3.5) \\
\end{array}$ & $\begin{array}{l}352 \\
(88.0) \\
\end{array}$ & $\begin{array}{l}17 \\
(4.3)\end{array}$ & $\begin{array}{l}31 \\
(7.7) \\
\end{array}$ \\
\hline $\begin{array}{l}\text { Spreading } \\
\text { awareness about } \\
\text { adverse effects }\end{array}$ & $\begin{array}{l}172 \\
(86.0)\end{array}$ & $\begin{array}{l}4 \\
(2.0)\end{array}$ & $\begin{array}{l}24 \\
(12.0)\end{array}$ & $\begin{array}{l}170 \\
(85.0)\end{array}$ & $\begin{array}{l}21 \\
(10.5)\end{array}$ & $\begin{array}{l}9 \\
(4.5)\end{array}$ & $\begin{array}{l}342 \\
(85.5)\end{array}$ & $\begin{array}{l}25 \\
(6.2)\end{array}$ & $\begin{array}{l}33 \\
(8.3)\end{array}$ \\
\hline
\end{tabular}

Table 3: Association of students practicing self-medication and various factors.

\begin{tabular}{|c|c|c|c|c|c|c|c|c|c|}
\hline \multirow[b]{3}{*}{ Characteristics } & \multirow{3}{*}{ 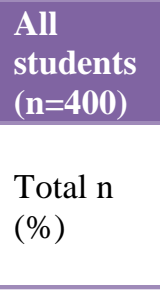 } & \multicolumn{8}{|c|}{ Students practicing self-medication } \\
\hline & & \multicolumn{4}{|c|}{ Medical $(n=400)$} & \multicolumn{4}{|c|}{ Nursing $(n=400)$} \\
\hline & & $\begin{array}{l}\text { Yes } \\
\mathrm{n}(\%)\end{array}$ & $\begin{array}{l}\text { No } \\
\mathrm{n}(\%)\end{array}$ & $\begin{array}{l}\text { Total } \\
\mathrm{n}(\%)\end{array}$ & $\begin{array}{l}\chi^{2} \\
\text { value, } \\
\mathrm{p} \\
\text { value }\end{array}$ & $\begin{array}{l}\text { Yes } \\
\mathrm{n}(\%)\end{array}$ & $\begin{array}{l}\text { No } \\
\mathrm{n}(\%)\end{array}$ & $\begin{array}{l}\text { Total } \\
\mathrm{n}(\%)\end{array}$ & $\begin{array}{l}\chi^{2} \\
\text { value, } \\
\mathrm{p} \\
\text { value }\end{array}$ \\
\hline \multicolumn{10}{|l|}{ Age (in years) } \\
\hline$<22$ years & $206(51.5)$ & $116(89.9)$ & $13(10.1)$ & $\begin{array}{l}129(100.0) \\
(64.5)\end{array}$ & \multirow{2}{*}{$\begin{array}{l}1.163 \\
0.281\end{array}$} & $66(85.7)$ & $11(14.3)$ & $\begin{array}{l}77(100.0) \\
(38.5)\end{array}$ & \multirow{2}{*}{$\begin{array}{l}0.050 \\
0.823\end{array}$} \\
\hline$\geq 22$ years & $\begin{array}{l}194 \\
(48.5)\end{array}$ & $67(94.4)$ & $4(5.6)$ & $\begin{array}{l}71(100.0) \\
(35.5)\end{array}$ & & 104(84.6) & $19(15.4)$ & $\begin{array}{l}123(100.0) \\
(61.5)\end{array}$ & \\
\hline \multicolumn{10}{|l|}{ Gender } \\
\hline Male & $\begin{array}{l}160 \\
(40.0)\end{array}$ & $105(89.7)$ & $\begin{array}{l}12 \\
(10.3)\end{array}$ & $\begin{array}{l}117(100.0) \\
(59.0)\end{array}$ & \multirow{2}{*}{$\begin{array}{l}1.118 \\
0.290\end{array}$} & $35(83.3)$ & $7(16.7)$ & $\begin{array}{l}42(100.0) \\
(21.0)\end{array}$ & \multirow{2}{*}{$\begin{array}{l}0.116 \\
0.734\end{array}$} \\
\hline Female & $\begin{array}{l}240 \\
(60.0)\end{array}$ & $78(94.0)$ & $5(6.0)$ & $\begin{array}{l}83(100.0) \\
(41.0)\end{array}$ & & $135(85.4)$ & $23(14.3)$ & $\begin{array}{l}158(100.0) \\
(79.0)\end{array}$ & \\
\hline \multicolumn{10}{|l|}{ Religion } \\
\hline Hindu & $\begin{array}{l}374 \\
(93.5)\end{array}$ & $175(92.1)$ & $15(7.9)$ & $\begin{array}{l}190(100.0) \\
(95.0)\end{array}$ & \multirow{4}{*}{$\begin{array}{l}7.410 \\
0.050\end{array}$} & $\begin{array}{l}155 \\
(84.2)\end{array}$ & $\begin{array}{l}29 \\
(15.8)\end{array}$ & $\begin{array}{l}184 \\
(100.0) \\
(92.0)\end{array}$ & \multirow{4}{*}{$\begin{array}{l}0.735 \\
0.885^{*}\end{array}$} \\
\hline Muslim & $8(3.0)$ & $2(50.0)$ & $2(50.0)$ & $\begin{array}{l}4(100.0) \\
(2.0)\end{array}$ & & $7(87.5)$ & $1(12.5)$ & $\begin{array}{l}8(100.0) \\
(4.0)\end{array}$ & \\
\hline Christian & $9(2.3)$ & $3(100.0)$ & 0 & $\begin{array}{l}3(100.0) \\
(1.5)\end{array}$ & & $6(3.5)$ & 0 & $\begin{array}{l}6(100.0) \\
(3.0)\end{array}$ & \\
\hline Buddhist & $5(1.2)$ & $3(100.0)$ & 0 & $3(100.0)$ & & $2(100.0)$ & 0 & $2(100.0)$ & \\
\hline
\end{tabular}




\begin{tabular}{|c|c|c|c|c|c|c|c|c|c|}
\hline & & & & $(1.5)$ & & & & $(1.0)$ & \\
\hline \multicolumn{9}{|l|}{ Caste } & \\
\hline General & $204(51.0)$ & $110(94.8)$ & $6(5.2)$ & $\begin{array}{l}116(100.0) \\
(58.0)\end{array}$ & \multirow{4}{*}{$\begin{array}{l}5.060 \\
0.136 *\end{array}$} & $79(89.8)$ & $9(10.2)$ & $\begin{array}{l}88(100.0) \\
(44.0)\end{array}$ & \multirow{4}{*}{$\begin{array}{l}9.522, \\
0.023\end{array}$} \\
\hline $\mathrm{OBC}$ & $77(19.3)$ & 29 (87.9) & $4(12.1)$ & $\begin{array}{l}33(100.0) \\
(16.5)\end{array}$ & & $34(77.3)$ & $10(22.7)$ & $\begin{array}{l}44(100.0) \\
(22.0)\end{array}$ & \\
\hline $\mathrm{SC}$ & 79 (19.7) & $26(83.9)$ & $5(16.1)$ & $\begin{array}{l}31(100.0) \\
(15.5)\end{array}$ & & $37(77.1)$ & 11(22.9) & $\begin{array}{l}48(100.0) \\
(24.0)\end{array}$ & \\
\hline ST & $40(10.0)$ & $18(90.0)$ & $2(10.0)$ & $\begin{array}{l}20(100.0) \\
(10.0)\end{array}$ & & $20(100.0)$ & 0 & $\begin{array}{l}20(100.0) \\
(10.0)\end{array}$ & \\
\hline \multicolumn{9}{|l|}{ Academic year } & \multirow{5}{*}{$\begin{array}{l}2.039 \\
0.564\end{array}$} \\
\hline $1^{\text {st }}$ year & $\begin{array}{l}100 \\
(25.0)\end{array}$ & $43(86.0)$ & $7(14.0)$ & $\begin{array}{l}50(100.0) \\
(25.0)\end{array}$ & \multirow{4}{*}{$\begin{array}{l}3.572 \\
0.327 *\end{array}$} & $40(80.0)$ & $\begin{array}{l}10 \\
(20.0)\end{array}$ & $\begin{array}{l}50(100.0) \\
(25.0)\end{array}$ & \\
\hline $2^{\text {nd }}$ year & $\begin{array}{l}100 \\
(25.0)\end{array}$ & $45(90.0)$ & $5(10.0)$ & $\begin{array}{l}50(100.0) \\
(25.0)\end{array}$ & & $45(90.0)$ & $5(10.0)$ & $\begin{array}{l}50(100.0) \\
(25.0)\end{array}$ & \\
\hline $3^{\text {rd }}$ year & $\begin{array}{l}100 \\
(25.0) \\
\end{array}$ & $47(94.0)$ & $3(6.0)$ & $\begin{array}{l}50(100.0) \\
(25.0)\end{array}$ & & $42(84.0)$ & $8(16.0)$ & $\begin{array}{l}50(100.0) \\
(25.0)\end{array}$ & \\
\hline $4^{\text {th }}$ year & $\begin{array}{l}100 \\
(25.0)\end{array}$ & $48(96.0)$ & $2(4.0)$ & $\begin{array}{l}50(100.0) \\
(25.0)\end{array}$ & & $43(86.0)$ & 7 (14.0) & $\begin{array}{l}50(100.0) \\
(25.0)\end{array}$ & \\
\hline \multicolumn{10}{|l|}{ Type of family } \\
\hline Nuclear & $\begin{array}{l}328 \\
(82.0)\end{array}$ & $\begin{array}{l}161 \\
(92.0)\end{array}$ & $14(8.0)$ & $\begin{array}{l}175 \\
(100.0) \\
(87.5)\end{array}$ & \multirow{2}{*}{$\begin{array}{l}0.410 \\
0.502 *\end{array}$} & $\begin{array}{l}132 \\
(86.3)\end{array}$ & $\begin{array}{l}21 \\
(13.7)\end{array}$ & $\begin{array}{l}153 \\
(100.0) \\
(76.5)\end{array}$ & \multirow{2}{*}{$\begin{array}{l}0.829 \\
0.362\end{array}$} \\
\hline Joint & $72(18.0)$ & $22(88.0)$ & $3(12.0)$ & $\begin{array}{l}25(100.0) \\
(12.5)\end{array}$ & & $38(80.9)$ & $9(19.1)$ & $\begin{array}{l}47(100.0) \\
(23.5)\end{array}$ & \\
\hline \multicolumn{10}{|c|}{ Socio-economic status (Modified BG Prasad Scale for 2017) } \\
\hline $\begin{array}{l}\text { Lower class } \\
(\mathrm{PCI}<938)\end{array}$ & $2(0.5)$ & $1(100.0)$ & 0 & $\begin{array}{l}1(100.0) \\
(0.5)\end{array}$ & \multirow{5}{*}{$\begin{array}{l}2.426 \\
0.829 *\end{array}$} & $1(100.0)$ & 0 & $\begin{array}{l}1(100.0) \\
(0.5)\end{array}$ & \multirow{5}{*}{$\begin{array}{l}2.455 \\
0.630 *\end{array}$} \\
\hline $\begin{array}{l}\text { Lower middle } \\
\text { class }(\text { PCI } \\
=938-1875)\end{array}$ & $6(1.5)$ & $1(100.0)$ & 0 & $\begin{array}{l}1(100.0) \\
(0.5)\end{array}$ & & $5(100.0)$ & 0 & $\begin{array}{l}5(100.0) \\
(2.5)\end{array}$ & \\
\hline $\begin{array}{l}\text { Middle Class } \\
(\mathrm{PCI}=1875- \\
3126)\end{array}$ & $30(7.5)$ & $4(100.0)$ & 0 & $\begin{array}{l}4(100.0) \\
(2.0)\end{array}$ & & $22(84.6)$ & $4(15.4)$ & $\begin{array}{l}26(100.0) \\
(13.0)\end{array}$ & \\
\hline $\begin{array}{l}\text { Upper middle } \\
\text { class }(\mathrm{PCI}= \\
3127-6253)\end{array}$ & $98(24.5)$ & $23(88.5)$ & $3(11.5)$ & $\begin{array}{l}26(100.0) \\
(13.0)\end{array}$ & & $58(80.6)$ & $\begin{array}{l}14 \\
(19.4)\end{array}$ & $\begin{array}{l}72(100.0) \\
(36.0)\end{array}$ & \\
\hline $\begin{array}{l}\text { Upper class } \\
(\mathrm{PCI} \geq 6254)\end{array}$ & $\begin{array}{l}264 \\
(66.0)\end{array}$ & $\begin{array}{l}154 \\
(91.7)\end{array}$ & $14(8.3)$ & $\begin{array}{l}168 \\
(100.0) \\
(84.0)\end{array}$ & & $84(87.5)$ & $12(12.5)$ & $\begin{array}{l}96(100.0) \\
(48.0)\end{array}$ & \\
\hline \multicolumn{10}{|l|}{ Attitude } \\
\hline $\begin{array}{l}\text { Negative } \\
(\leq \text { median } \\
\text { value })\end{array}$ & $\begin{array}{l}231 \\
(57.8)\end{array}$ & $\begin{array}{l}102 \\
(95.3)\end{array}$ & $5(4.7)$ & $\begin{array}{l}107 \\
(100.0) \\
(53.5)\end{array}$ & & $106(85.5)$ & $18(14.5)$ & $\begin{array}{l}124(100.0) \\
(62.0)\end{array}$ & \multirow[b]{2}{*}{$\begin{array}{l}0.060 \\
0.807\end{array}$} \\
\hline $\begin{array}{l}\text { Positive } \\
\text { (>median } \\
\text { value) } \\
\text { (median value= } \\
19 \text { and } \\
20 \text { for medical } \\
\text { and nursing } \\
\text { students } \\
\text { respectively }\end{array}$ & $\begin{array}{l}169 \\
(42.2)\end{array}$ & $\begin{array}{l}81 \\
(87.1)\end{array}$ & $\begin{array}{l}12 \\
(12.9)\end{array}$ & $\begin{array}{l}93(100.0) \\
(46.5)\end{array}$ & $\begin{array}{l}4.333 \\
0.037\end{array}$ & $64(84.2)$ & $\begin{array}{l}12 \\
(15.8)\end{array}$ & $\begin{array}{l}76(100.0) \\
(38.0)\end{array}$ & \\
\hline Total & $400(100.0)$ & $\begin{array}{l}183 \\
(91.5)\end{array}$ & $17(8.5)$ & $\begin{array}{l}200 \\
(100.0)\end{array}$ & & $\begin{array}{l}170 \\
(85.0)\end{array}$ & $\begin{array}{l}30 \\
(15.0)\end{array}$ & $\begin{array}{l}200 \\
(100.0)\end{array}$ & \\
\hline
\end{tabular}


Table 4: Findings of focus group discussion to explore relation of Knowledge, Attitude and Practice of Self Medication among undergraduate medical and B. Sc nursing students.

\begin{tabular}{|c|c|c|}
\hline Category & Code & Interpretation \\
\hline \multirow{6}{*}{ Self-medication } & Definition & $\begin{array}{l}\text { Self-medication is the treatment of common ailments with medicines that are taken by } \\
\text { patient's own choice without doctor's advice. }\end{array}$ \\
\hline & $\begin{array}{l}\text { Source of } \\
\text { knowledge }\end{array}$ & $\begin{array}{l}\text { They got Lecture classes, Internet, friends/ seniors, old prescriptions, pharmacist, } \\
\text { magazine, newspaper, mass media, guardian. }\end{array}$ \\
\hline & $\begin{array}{l}\text { Knowledge of } \\
\text { medication }\end{array}$ & $\begin{array}{l}\text { Majority medical students know about the dosage and side effects of drugs, hazards due } \\
\text { to increase drug dose and due to change of timing, in comparison to nursing students. }\end{array}$ \\
\hline & \multirow{3}{*}{$\begin{array}{l}\text { Source of } \\
\text { drugs }\end{array}$} & Medicine shop is the commonest place to buy medicines for self-medication. \\
\hline & & Few collect in from friends/ seniors, relatives, neighbours, guardians. \\
\hline & & $\begin{array}{l}\text { 'Sometimes we get free medicine samples from our seniors (internees and junior } \\
\text { residents) in hostel. We keep those with us, use as and when required' }\end{array}$ \\
\hline \multirow{7}{*}{$\begin{array}{l}\text { Self-medication } \\
\text { pattern }\end{array}$} & \multirow[b]{2}{*}{$\begin{array}{l}\text { Medication } \\
\text { use }\end{array}$} & They use for themselves, family members, relatives, friends, neighbours. \\
\hline & & $\begin{array}{l}\text { "Friends/ relatives often ask me to tell them about few medicine name for gas/ GI upset, } \\
\text { headache" }\end{array}$ \\
\hline & \multirow{2}{*}{$\begin{array}{l}\text { Type of } \\
\text { medicine }\end{array}$} & $\begin{array}{l}\text { Majority students use Allopathic medicine, few use Ayurvedic and Homoeopathic } \\
\text { medicine. }\end{array}$ \\
\hline & & $\begin{array}{l}\text { Only very few students use herbal products as seen in TV advertisement, magazine, } \\
\text { newspaper. }\end{array}$ \\
\hline & Disease & $\begin{array}{l}\text { Medication is used for common ailments such as fever, headache, abdominal pain, } \\
\text { gastritis, vomiting, diarrhoea, common cold and cough, skin problems, rash, allergies, } \\
\text { muscular/ joint pain. }\end{array}$ \\
\hline & Drugs & $\begin{array}{l}\text { They use variety of medicines like analgesic, antipyretics, coughs syrup, antihistaminic, } \\
\text { antiemetic, multi- vitamins, antispasmodic, topical preparation; eye/ ear drops, herbal } \\
\text { preparation. }\end{array}$ \\
\hline & Duration & $\begin{array}{l}\text { Majority students stop the drugs when the symptoms disappear whether course completed } \\
\text { or not; few of them, take the drug on and off. }\end{array}$ \\
\hline \multirow{8}{*}{$\begin{array}{l}\text { Influencing } \\
\text { factors }\end{array}$} & $\begin{array}{l}\text { Reason for } \\
\text { self- } \\
\text { medication }\end{array}$ & $\begin{array}{l}\text { Prior experience, Non-seriousness illness, Emergency use, seeking quick relief, Time- } \\
\text { saving, Cost-effectiveness is some of the reason which leads them to practice self } \\
\text { medication. }\end{array}$ \\
\hline & \multirow{3}{*}{$\begin{array}{l}\text { Reasons for } \\
\text { not consulting } \\
\text { a doctor }\end{array}$} & "I am confident enough to take medicines for minor illness" \\
\hline & & $\begin{array}{l}\text { Minor illness, lack of time to consult doctor, not willing to spend money in doctor's fee } \\
\text { and doctors may suggest laboratory investigations. }\end{array}$ \\
\hline & & $\begin{array}{l}\text { "Unavailability of health-care facilities nearby my home, so I keep few medicines } \\
\text { (abdominal pain, diarrhoea) in my home which may be needed in odd hours" }\end{array}$ \\
\hline & \multirow{4}{*}{$\begin{array}{l}\text { Contributory } \\
\text { factors }\end{array}$} & "Who will go to a doctor for simple fever, headache or abdominal pain?" \\
\hline & & $\begin{array}{l}\text { "Our teachers often ask subject related questions when we go to them for own illness. So } \\
\text { I often took medicine on my own for minor illness." }\end{array}$ \\
\hline & & $\begin{array}{l}\text { They use previous prescription when experienced similar symptoms and even give that } \\
\text { prescription to someone who is having similar symptoms. }\end{array}$ \\
\hline & & Easy availability of medicines from medicine shop, seniors in hostel. \\
\hline \multirow{3}{*}{ Health hazards } & \multirow{2}{*}{ Side effects } & They have experienced side effects like drowsiness (cetrizine), nausea etc. \\
\hline & & Medicine was stopped when side effects appeared. \\
\hline & Experience & $\begin{array}{l}\text { Though there are some side effects, still they get benefitted from it and advise this to } \\
\text { friends and peer groups, relatives. }\end{array}$ \\
\hline \multirow{7}{*}{ Awareness } & \multirow[b]{2}{*}{ Opinion } & Self-medication is acceptable for medical students, not others. \\
\hline & & $\begin{array}{l}\text { Though self medication is a part of self care but harmful effects on health are there. } \\
\text { "Thinking, if my peers stop, I will stop self medication" }\end{array}$ \\
\hline & $\begin{array}{l}\text { Reason } \\
\text { against self- } \\
\text { medication }\end{array}$ & $\begin{array}{l}\text { There are chances of missing actual diagnosis; side effects, drug dependence, not taking } \\
\text { complete dose and course (particularly in case of antibiotics) of therapy. }\end{array}$ \\
\hline & \multirow{4}{*}{$\begin{array}{l}\text { Preventive } \\
\text { strategies }\end{array}$} & Newly admitted Student should be informed \& educated. \\
\hline & & $\begin{array}{l}\text { "Government should bring rules against advertisement and sell of pharmaceutical } \\
\text { products, health supplements without prescription" }\end{array}$ \\
\hline & & $\begin{array}{l}\text { 'We can make people aware about possible health hazards of self medication and making } \\
\text { health care facilities easily available, but still it remain a personal choice' }\end{array}$ \\
\hline & & $\begin{array}{l}\text { 'Spreading awareness about emergence of drug resistance, when antibiotic dose is left in } \\
\text { midway after 2-3 days when he/she feels well'. }\end{array}$ \\
\hline
\end{tabular}


Total attitude score was calculated, mean was 19.1 \pm 2.9 and $19.1 \pm 2.7$ for medical and nursing students respectively.

Table 3 showed that study participants (both medical and nursing students) aged 22years old or above, female, Hindu religion, general/OBC/ST community, higher academic year $\left(3^{\text {rd }}\right.$ and $4^{\text {th }}$ year followed by $2^{\text {nd }}$ year in each stream), those belonging to nuclear families, upper class and upper middle class, negative attitude towards self-medication had more practice of self-medication compared to their counterparts. Statistically significant $(\mathrm{P}<0.05)$ association of self-medication practices was found with religion and attitude in case of medical students and caste of nursing students respectively (Table 3).

Various issues related to self-medication were merged under 17 codes from the transcripts, which were grouped into five categories. Five categories were- ideas of selfmedication and its pattern, influencing factors, health hazards, awareness. These, along with responses provided by the respondents regarding self-medication were listed as interpretation (Table 4).

\section{DISCUSSION}

In this study among 400 students (200 medical and 200 nursing students), majority $(64.5 \%)$ medical students were $<22$ years old (mean age $20.9 \pm 1.3$ years) whereas $61.5 \%$ nursing students were $\geq 22$ years (mean age 22.0 \pm 2.0 years), almost similar to the findings of other studies., ${ }^{79,21}$ Philip PT et al, in Tamil Nadu showed that maximum (29.14\%) respondents were 20 years old followed by $26.34 \%$ in 19years. ${ }^{6}$ Few studies included participants from various professional education, i.e BN, Bsc Nursing, BDS, MBBS and B. Pharmacy, physiotherapy, Paramedical. ${ }^{7,21,22}$ Overall, female predominance $(60 \%)$ was seen in present study (59\% male among medical, whereas $79 \%$ female among nursing students) similar to other studies. ${ }^{4,6,21}$ Dutta $S$ et al, found more male students $\left(59.07 \%\right.$ in medical vs $47.79 \%$ in non-medical courses). ${ }^{19}$ Johnson D et al, and Mehta RK et al, found higher (70.1\% and $72 \%$ respectively) female participation than present study. ${ }^{7,22}$

Present study found that overall $88.3 \%$ (91.5\% medical vs $85 \%$ nursing) students practiced self-medication which was consistent with studies done elsewhere. ${ }^{4,6,7,21}$ Dutta $S$ et al, found overall less $(67 \%)$ of self-medication, but more $(71 \%)$ medical students practiced than $(63 \%)$ non-medical students $(\mathrm{p}<0.05) .{ }^{19}$ Johnson D et al, found slightly more self-medication (92.39\%) with higher practice among pharmacy $(92.35 \%)$ and nursing $(99.4 \%)$ than medical students $(86.3 \%) .^{22}$

Philip PT et al, found that $31.33 \%$ students recommended drugs to their families and friends. ${ }^{6}$ Qualitative analysis in present study found that students practiced for themselves, family members, relatives, friends, neighbours; similar to other studies. ${ }^{4,7}$ Students in present study also stated that
"Friends/ relatives often ask me to tell them about few medicine names for gas/ GI upset, headache".

Similar to present study; textbook was reported as their primary source of information followed by parents, friends, old prescriptions, internet, pharmacist in some other studies. ${ }^{4,6,7,21}$ Dutta $S$ et al, found that the most common source of information for medicos are textbooks $(67.5 \%)$ and physician or pharmacists $(66 \%)$ whereas for nonmedical students, pharmacist $(68 \%)$ and previous prescriptions $(66 \%)$ were most common sources. ${ }^{19}$

Johnson D et al, found that pharmacy was their source of drug (96.9\%); others were parents $(27.8 \%)$, friends $(15.3 \%)$, relatives $(8.28 \%)$ and herbal store $(6.9 \%){ }^{22}$ Present qualitative analysis found medicine shop as the commonest place to buy medicines; few collect from friends/seniors, relatives, neighbours, guardians. Philip PT et al, revealed that $70.80 \%$ obtained drugs directly from medical shop; others use leftover medicines, hospital supplies, physician samples etc. ${ }^{6}$

Previous literature reported minor illness as main reason for self-medication; others were quick relief of symptoms, confident to treat, lack of time, financial constraints, and non-availability of health-care facilities. ${ }^{6,7,19,21,22}$

In present study, confidence and awareness about medicine (62.3\% medical vs $15.3 \%$ nursing students), lack of time to visit a doctor (7.6\% medical vs $41.2 \%$ nursing students), reluctance to spend money (5.5\% medical vs $12.9 \%$ nursing students), quick relief (22.4\% medical vs $10.6 \%$ nursing students) were reason behind self-medication. Dutta $\mathrm{S}$ et al, showed that awareness constitutes $56.89 \%$ in medical and $32 \%$ in non-medical students $(\mathrm{p}<0.0001) .{ }^{19}$ Majority (overall $40.2 \%$; $49.7 \%$ medical vs $30 \%$ nursing students) took self-medication having previous experience of disease whereas Damodar G et al, found $35.23 \%$ had previous experience of treating similar disease. ${ }^{4}$

Mehta RK et al, found that reason against self-medication were afraid of adverse drug reaction (50\%), wrong diagnosis (50\%), missing actual diagnosis (33.3\%) and drug dependence $(25 \%) .^{7}$ Present qualitative analysis revealed missing actual diagnosis, side effects, drug dependence, not taking complete dose and course (particularly in case of antibiotics) points against selfmedication.

Mehta RK found that $85.7 \%$ use medication for cold and cough, $76.2 \%$ for pain; fever $(73.0 \%)$, diarrhea $(47.6 \%)$, dysmenorrhea (46.0\%) and nausea- vomiting (30.2\%). ${ }^{7}$ In our study majority (53\%) took self-medication for headache $(79.2 \%$ medical vs $72.4 \%$ nursing students) followed by fever $(77.6 \%$ medical vs $52.4 \%$ nursing students), (12.5\%), common cold $(72.7 \%$ medical vs $49.4 \%$ nursing students) consistent with other findings. ${ }^{6,21,22}$ 
Literature review revealed that most common drugs used were analgesics, antipyretics, anta-acids and antispasmodic. ${ }^{6,79-21}$ Damodar G et al, reported $88.08 \%$ used antibiotics and cough syrup followed by $74.09 \%$ analgesics for self-medication in the preceding 12 months. ${ }^{4}$ In present study most commonly used drugs were vitamins and antioxidants $(67.8 \%)$ followed by antibiotics $(61.2 \%)$ and cough syrup (55.9\%) and other drugs, i.e. analgesics (23.5\% medical vs $20.6 \%$ nursing students), antipyretics (42.6\% medical vs $34.7 \%$ nursing students), anti-allergies (7.6\% medical vs 5.9\% nursing students). Mehta RK et al, found that antibiotics $25(40.3 \%)$, vitamins $23(37.1 \%)$, anti-allergies $22(35.5 \%)$ and herbal $19(30.6 \%)$ were also used. $^{7}$

Mehta RK et al, found that $36 \%$ had some knowledge on drugs adverse reaction and $25.3 \%$ had little knowledge on completing dose. ${ }^{7}$ Present study found that majority medical students $(70.5 \%)$ as compared to nursing students $(50.6 \%)$ knew about side effects and majority $(58.5 \%$ medical vs $45.3 \%$ nursing students) had withdrawn medication after symptoms were over. Philip PT et al, found that $57.21 \%$ students completed course and $23.94 \%$ were aware of ADRs. ${ }^{6}$ Dutta S et al, found that $46 \%$ medical students complete full treatment as compared to $32 \%$ non-medical students and maximum students (93\%) read label instructions as compared to present study $(67.4 \%) .{ }^{19}$ More than half $(55.8 \%)$ of the participants (66.7\% medical vs $44.12 \%$ nursing) completed full course of therapy whereas $37.82 \%$ students for 3-5 days and $17 \%$ students for $>5$ days as shown by Damodar G et al. ${ }^{4}$

Singla S et al, and Philip PT et al, found that $6 \%$ and $19.71 \%$ students respectively experienced side effect, in present study majority nursing students (52.9\%) experienced adverse effects than medical students $(13.5 \%) .^{6,21}$ Dutta $\mathrm{S}$ et al, found on and off drug use pattern (33\% medical vs. $63 \%$ in non-medical students), with stoppage of drug on appearance of adverse symptoms (21\% of medical vs $5 \%$ of non-medical students). ${ }^{19}$ Present study showed that majority students stop taking drug when symptoms disappear and when side effects appeared; few of them take the drug on and off as and when required. Mehta RK et al, found that $61.3 \%$ didn't discontinue medicines when symptoms were not relieved, $72 \%$ didn't experience adverse reaction and $88 \%$ won't habitual to any drug. ${ }^{7}$ Qualitative analysis found that majority students opined though there are some side effects; still they got benefitted and advise this to friends and peer groups, relatives. Johnson D et al, found that $94 \%$ benefited from self-medications. ${ }^{22}$ Philip PT et al, found that only $18.18 \%$ students use alternative medicines like Ayurveda, Homeopathy etc., without medical advice. ${ }^{6}$ In present study maximum respondents preferred allopathic medicine ( $87.4 \%$ medical vs $85.9 \%$ nursing students) while majority nursing students used Homoeopathy and Ayurveda; similar to more use of Ayurveda by non-medical students. ${ }^{19}$

Dutta $\mathrm{S}$ et al, showed that $85 \%$ non-medical students didn't know whether self-medication was correct or not. ${ }^{19}$ Mehta
RK et al, found higher $(50.7 \%)$ positive attitude than present study $(42.2 \%) .{ }^{7}$ Present study found that less than half of the respondents $(48.5 \%$ medical vs $46.5 \%$ nursing students) believed self-medication as harmful, whereas $71.5 \%$ nursing students believed this as self-care as compared to medical students $(56.5 \%)$; when asked about continuation, only $21 \%$ nursing and $32.5 \%$ medical students reported to continue in future also. Damodar $\mathrm{G}$ et al, found most of them $(80.94 \%)$ disagree with selfmedication practices. ${ }^{4}$ Johnson $\mathrm{D}$ et al, found, $28.2 \%$ pharmacy students believed this as a self-care but $5.4 \%$ medical professionals didn't believed so. ${ }^{22}$ Similarly, $42.1 \%$ of students told that they will continue/start selfmedication practice (mostly medical) whereas $20.24 \%$ told that they won't continue/start (mostly nurses); while, $47.14 \%$ students replied advising to their friends in near future. ${ }^{22}$

Opinion in present study to prevent self-medication revealed consistent findings with Johnson D et al, where students suggested to promote awareness and education regarding self-medication, making health care facilities easily available, to enforce strict rule regarding misleading pharmaceutical advertisement but few supported to prevent supply of medicine from pharmacy. ${ }^{22}$

Damodar $\mathrm{G}$ et al, found no significant difference between self-medication practices of medicine, pharmacy and nursing students; male and female and year of study ( $p$ $>0.05$ ) matched with present study findings. ${ }^{4}$ Present study also found significant ( $\mathrm{P}<0.05)$ association of selfmedication with religion and attitude in case of medical students and caste of nursing students respectively.

\section{CONCLUSION}

Self-medication was widely practiced among students for minor illness, lack of health services and easy availability of drugs. Making healthcare system easily available and promoting education regarding self-medication can reduce the risk. A separate module on pros and cons of selfmedication and drug-induced injury in professional teaching curriculum is a need of hour. Responsible selfmedication being safe and effective for common illnesses not requiring medical consultation can be a cheaper alternative and will reduce burden on Indian health care system. Lastly, government support in curbing easy availability of medicine across pharmacies can also reduce self-medication practices substantially.

\section{ACKNOWLEDGEMENTS}

The authors would like to thank all the participants for giving their valuable time in conduction of this research study.
Funding: No funding sources
Conflict of interest: None declared
Ethical approval: The study was approved by the Institutional Ethics Committee 


\section{REFERENCES}

1. Pereira CM, Alves VF, Gasparetto PF, Carneiro DS, Carvalho DD, Valoz FE. Self-medication in health students from two Brazilian universities. RSBO (Online). 2012 Dec;9(4):361-7.

2. Kumari R, Kiran KD, Bahl R, Gupta R. Study of knowledge and practices of self-medication among medical students at Jammu. J Med Sci. 2012;15(2):141-4.

3. The Role of the Pharmacist in Self-Care and SelfMedication. Essential Medicines and Health Products Information Portal. World Health Organization Resource; 1998. Available at: http://apps.who.int/ medicinedocs/en/d/Jwhozip32e/3.2.html Accessed 12 January 2018.

4. Damodar G. Assessment of self-medication practices among medical, pharmacy and nursing students at a tertiary care teaching hospital. IJHP. 2012;49:79-83.

5. Adhikary M, Tiwari P, Singh S, Karoo C. Study of self-medication practices and its determinants among college students of Delhi university north campus, New Delhi, India. Int J Med Sci Public Health. 2014;3(4):406-9.

6. Philip PT, Senan A, Reji S, Kumar TA. Assessment of self medication practices among undergraduate medical and paramedical students: A Case of rural Medical Schools of Tamil Nadu, India. World J Pharmacy Pharmaceut Sci. 2015 Aug 8;4(10):1587604.

7. Mehta RK, Sharma s. Knowledge, Attitude and Practice of Self-Medication among Medical Students. IOSR J Nursing Health Sci. 2015;4(1):89-96.

8. Pandya RN, Jhaveri KS, Vyas FI, Patel VJ. Prevalence, pattern and perceptions of self-medication in medical students. Int $\mathbf{J}$ Basic Clin Pharmacol. 2013;2(3):275-80.

9. Shankar PR, Partha P, Shenoy N. Self-medication and non-doctor prescription practices in Pokhara valley, Western Nepal: a questionnaire-based study. BMC Family Practice. 2002 Dec;3(1):17.

10. Walia JK. Over the Counter Sale of Drugs- How to Curb this Practice? India Medical Times; 2012. Available at: http://www. indiamedicaltimes.com/2012/11/13/over-the-countersale-of-drugshow-to-curb-this-practice/. Accessed on January 2018.

11. Kumarasamy KK, Toleman MA, Walsh TR, Bagaria J, Butt F, Balakrishnan R, et al. Emergence of a new antibiotic resistance mechanism in India, Pakistan, and the UK: A molecular, biological, and epidemiological study. Lancet Infect Dis. 2010;10:597-602.
12. IHS, SSK, Jayan M, Hussain A. Prevalence of self medication practices and its associated factors in rural Bengaluru, Karnataka, India. Int J Community Med Public Health. 2016;3(6):1481-6.

13. Banerjee I, Bhadury T. Self-medication practice among undergraduate medical students in a tertiary care medical college, West Bengal. J Postgrad Med. 2012 Apr 1;58(2):127.

14. Badiger S, Kundapur R, Jain A, Kumar A, Pattanshetty S, Thakolkaran N, et al. Self-medication patterns among medical students in South India. Australasian Med J. 2012;5(4):217-20.

15. Simon AK, Rao A, Rajesh G, Shenoy R, Pai MB. Trends in self-medication for dental conditions among patients attending oral health outreach programs in coastal Karnataka, India. Indian J Pharmacol. 2015 Sep;47(5):524-9.

16. Montgomery AJ, Bradley C, Rochfort A, Panagopoulou E. A review of self-medication in physicians and medical students. Occup Med. $2011 \mathrm{Jul}$ 4;61(7):490-7.

17. Alano GM, Galafassil LM, Galato D, Trauthman SC. Responsible for self medication review of the process of pharmaceutical attendance. Braz J Pharm Sci. 2009;45(4):626-33.

18. James H, Handu SS, Al Khaja KA, Otoom S, Sequeira RP. Evaluation of the knowledge, attitude and practice of self-medication among first-year medical students. Med Principles Pract. 2006;15(4):270-5.

19. Dutta S, Hazarika K. Pattern of self-medication and drugs use behavior among undergraduate students of medical and non-medical colleges in a city of NorthEast India - A Comparative Study. Asian J Pharmaceut Clin Res. 2016; 6(9):259-62.

20. Singh T, Sharma S, Nagesh S. Socio-economic status scales updated for 2017. Int J Res Med Sci. 2017;5(7):3264-7.

21. Singla S, Kaur M, Mahajan R. Pattern and practice of self medication in students of health professional university in North India. Int J Basic Clin Pharmacol. 2017 Oct 25;6(11):2607-11.

22. Johnson DE, Sekhar HS, Alex TE, Kumaraswamy M, Chopra RS. Self medication practice among medical, pharmacy and nursing students. Int $\mathbf{J}$ Pharm Pharmaceut Sci. 2016;8(7):1-5.

Cite this article as: Karmakar N, Chakraborty T, Datta A, Nag K, Datta SS. Self-medication practices among medical and nursing students in Tripura, India: a mixed-method study. Int J Basic Clin Pharmacol 2019;8:296-305. 